\title{
Indicativos do processo de avaliação de serviços de Saúde Mental*
}

Indicators of mental health services evaluation process

Indicadores del proceso de evaluación de servicios de Salud Mental

Ana Caroline Gonçalves Cavalcante ${ }^{1}$, Elizabeth Esperidião², Nathália Santos Silva ${ }^{3}$, Kellykan Silva Silva ${ }^{4}$, Adrielle Cristina Silva Souza ${ }^{5}$

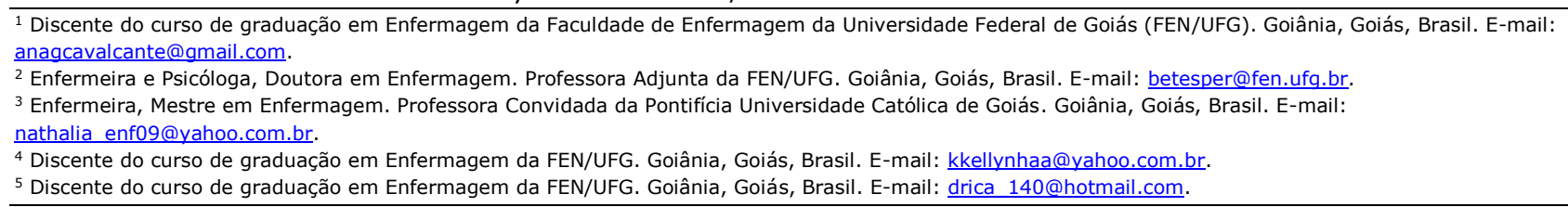

\section{RESUMO}

Estudo descritivo exploratório de abordagem qualitativa que teve por objetivo avaliar a estrutura da Rede Especializada em Saúde Mental da Secretaria Municipal de Saúde de Goiânia, Goiás. Os dados, coletados por instrumento semiestruturado e registro fotográfico, foram analisados com auxílio do software Atlas.ti 6.2 e com base no referencial teórico de Donabedian. Encontrou-se variedade de condições na estrutura física dos serviços, desde precariedade das instalações e ausência de ambientes propícios à execução das práticas terapêuticas, até ambientes acolhedores com boa acessibilidade. Destaca-se como aspecto favorável a diversidade na composição das equipes multiprofissionais. É premente a adequação da estrutura dos serviços, embora se reconheça que o município está em franco processo de reformulação, na perspectiva de atender às necessidades da Política Nacional de Saúde Mental. Também para processos avaliativos, recomendam-se parcerias interinstitucionais, especialmente no âmbito da academia e dos serviços, que podem gerar o impacto desejado na assistência aos usuários dos serviços especializados.

Descritores: Avaliação de Serviços de Saúde; Saúde Mental; Estrutura dos Serviços.

\section{ABSTRACT}

This descriptive, exploratory and qualitative study was performed with the objective to evaluate the structure of the Mental Health Service Network of the Municipal Health Department of Goiania, the capital city of Goias state, Brazil. Data were collected using a semi-structured instrument and photographic records, and analyzed using Atlas.ti 6.2, and based on Donabedian's theoretical framework. Various conditions were observed for service facilities; from structures that were precarious and unsuitable for therapy, to facilities that were welcoming and had good accessibility. The main positive aspect was the diversity of multidisciplinary teams. Making service facilities appropriate is imperative, although it is recognized that the municipality is currently undergoing reformulation, aiming at meeting the needs of the National Policy for Mental Health. Furthermore, intersectoral partnerships should be established for evaluation processes, particularly in the academia and service domains, which could generate the desired impact on health care to clients of specialized services.

Descriptors: Health Services Evaluation; Mental Health; Structure of Services.

\section{RESUMEN}

Estudio descriptivo, exploratorio, de abordaje cualitativo, que objetivó evaluar la estructura de la Red Especializada en Salud Mental de la Secretaría Municipal de Salud de Goiânia-Goiás. Los datos, recolectados mediante instrumento semiestructurado y registro fotográfico, fueron analizados por software Atlas.ti 6.2 según referencial teórico de Donabedian. Se encontraron variadas condiciones en la estructura física de los servicios, desde precariedad de instalaciones, ausencia de ambientes propicios para ejecución de prácticas terapéuticas, hasta ámbitos acogedores con buena accesibilidad. Se destaca como aspecto favorable la diversidad en la composición de equipos multiprofesionales. Resulta apremiante la adecuación estructural de los servicios, aunque se reconoce que el municipio está en franco proceso de reformulación, apuntando a atender las necesidades de la Política Nacional de Salud Mental. También, para procesos evaluativos, se recomiendan alianzas inter-institucionales, especialmente con el ámbito académico y de servicios, que generen el deseado impacto en la atención de pacientes de servicios especializados.

Descriptores: Evaluación de Servicios de Salud; Salud Mental; Estructura de los Servicios. 


\section{INTRODUÇÃO}

A assistência em Saúde Mental passa por grandes mudanças em muitos países em que a desinstitucionalização, o desenvolvimento de serviços baseados na comunidade e a integração do sistema de saúde em geral são enfatizados(1). A proposta da especialidade nesta transição representa não apenas a transformação dos hospitais ou a ampliação da rede de serviços territoriais, mas o exercício de desinstitucionalizar a assistência, como potente ferramenta na luta pelo desmantelamento do sistema excludente manicomial e na construção de outras possibilidades assistenciais, terapêuticas e culturais ${ }^{(2)}$.

No Brasil, as lutas pela abertura política e por uma saúde pública de acesso universal caminharam conjuntamente na busca da assistência psiquiátrica mais humana e voltada para a melhoria da qualidade de vida por meio da ampliação das redes afetivas e sociais ${ }^{(3)}$.

Fundamentado em diversas experiências de reforma no atendimento psiquiátrico no mundo, o Ministério da Saúde brasileiro, a partir da década de 90, define uma proposta de atenção na área, que redireciona os recursos assistenciais para um modelo substitutivo, devidamente detalhado pela Política Nacional de Saúde Mental $\left(\right.$ PNSM $^{(4)}$.

A PNSM busca consolidar o modelo de atenção à saúde mental aberto e de base comunitária que garante a livre circulação das pessoas com transtornos mentais pelos serviços e comunidade oferecendo cuidados com base nos seus recursos sociais. Nesta perspectiva, a rede de serviços conta com os Centros de Atenção Psicossocial de diferentes tipos (CAPS), os Serviços Residenciais Terapêuticos (SRT), os Centros de Convivência e Cultura, leitos em Hospitais Gerais, Atenção Básica, dentre outros $^{(5)}$.

O surgimento destes serviços substitutivos, com princípios e tecnologias sensivelmente distintas das práticas psiquiátricas anteriores, trouxe ao debate na área da Saúde Mental a questão da necessidade de novos instrumentos de avaliação de qualidade dos serviços e das ações prestadas, uma vez que, os indicadores clássicos têm-se mostrado insuficientes ou inadequados ${ }^{(2)}$.

Sabe-se que os serviços são distribuídos desigualmente entre as regiões do país e ainda não há dados sólidos para mostrar o impacto da mudança política em termos de custo-efetividade(6).

Diante deste panorama, há recomendações de organismos internacionais de saúde pública (OPAS/OMS) de que os serviços de Saúde Mental sejam avaliados internacionalmente na tentativa de garantir a qualidade da assistência, bem como de alcançar as metas do movimento da Reforma Psiquiátrica no mundo(1). Para o Brasil, propõem que se contextualizem suas políticas públicas da área, além de avaliar o desempenho e a compreensão dos profissionais que nela atuam, tendo como referência a formação destes profissionais e a estrutura física dos serviços.

A literatura científica, aponta a diversidade dos aspectos relacionados à estrutura dos serviços de Saúde Mental, sinalizando dados quanto à organização, funcionamento e instalações físicas, além de equipes heterogêneas e a não padronização das atividades desenvolvidas. Estudos destacam a ampliação e adequação da ambiência e da estrutura física destes serviços, sendo componente importante no processo de trabalho e na qualificação da assistência oferecida(7-11).

A avaliação de serviços de Saúde Mental deve ser uma atividade contínua e processual, a fim de identificar aspectos assistenciais que necessitam ser reajustados com vistas à melhoria da terapêutica disponibilizada aos usuários e a qualidade dos serviços ${ }^{(1)}$.

Diante deste cenário, caracterizar a estrutura dos serviços de Saúde Mental é fundamental dentre os procedimentos de avaliação, na medida em que favorece a identificação das condições na prestação de atendimento, a qual tem como objeto privilegiado a ação social organizada com vistas a mudanças ${ }^{(11)}$.

Em se tratando de processos avaliativos, o referencial teórico de Donabedian ${ }^{(12)}$, adotado nesta investigação, prevê estrutura, processo e resultado como elementos chaves de análise. Em relação ao componente estrutura, considera aspectos alusivos à área de abrangência, estrutura física e recursos humanos.

Buscando atender tal demanda, este estudo, que integra um grande projeto de pesquisa em desenvolvimento no Estado de Goiás, financiado pelo CNPq e FAPEG (Fundação de Amparo à Pesquisa do Estado de Goiás) tem por objetivo avaliar a estrutura da Rede Especializada em Saúde Mental da Secretaria Municipal de Saúde de Goiânia-Goiás (RESM/SMS/GO).

Apontar indicativos do processo de avaliação da estrutura de serviços de Saúde Mental desta localidade, favorece o cumprimento das políticas públicas norteadoras da área, na medida em que dá visibilidade da realidade loco-regional contribuindo também para a reflexão do panorama brasileiro, resguardadas as devidas especificidades, além de fortalecer a parceria já existente entre os órgãos gestores da saúde municipal e estadual de Goiás e a Universidade na qual os pesquisadores estão vinculados. 


\section{METODOLOGIA}

Pesquisa descritiva exploratória de abordagem qualitativa desenvolvida em Goiânia, capital do Estado de Goiás, no período de janeiro a março de 2012.

A assistência em Saúde Mental nesta localidade é oferecida por meio de serviços privados, públicos e conveniados ao Sistema Único de Saúde (SUS). Deste modo, os portadores de transtorno mental e/ou usuários de álcool e outras drogas são atendidos tanto em hospitais/clínicas quanto em CAPS e ambulatórios.

O município de Goiânia está organizado em nove Distritos Sanitários, que por ocasião do desenvolvimento da pesquisa, dispunha de 16 serviços públicos de atendimento em Saúde Mental, em diferentes modalidades, constituindo a Rede Especializada em Saúde Mental (RESM/SMS/GO): um Pronto Socorro Psiquiátrico, sete CAPS, um Ambulatório de Psiquiatria, duas Unidades de Geração de Renda (Gerarte) e cinco Residências Terapêuticas.

Os serviços são distribuídos de acordo com suas respectivas áreas de abrangência e cabe à Divisão de Saúde Mental da Secretaria Municipal de Saúde (DSM/SMS/GO) a gestão, de forma a garantir a acessibilidade na rede às pessoas que necessitam de atenção especializada.

Considerando a especificidade e objetivos desta investigação, os dados foram coletados in loco nas 11 unidades que compunham a RESM/SMS/GO, exceto os serviços de Residência Terapêutica.

Utilizou-se um instrumento semiestruturado que norteou a observação dos aspectos a serem analisados, complementando-se por meio do registro fotográfico dos serviços visitados. O instrumento constituiu-se de um roteiro com questões que permitiram quantificar $e$ descrever o espaço físico de cada serviço, bem como os materiais e equipamentos neles alocados, além de identificar a área de abrangência, estrutura física e equipe envolvida. As fotografias possibilitaram maior detalhamento dos espaços, favorecendo a posterior análise.

Complementarmente à visita, alguns dados foram colhidos em consulta on line em documentos oficiais de Órgãos deliberativos de Políticas Públicas tais como Ministério da Saúde, Secretaria de Saúde do Estado de Goiás e o Departamento de Informática do SUS DATASUS.

O procedimento de análise seguiu-se pela apreciação das informações obtidas pelo instrumento e das imagens fotográficas, utilizando-se os recursos do software Atlas.ti 6.2 que contribuiu significativamente para a avaliação de aspectos estruturais, ao permitir a organização e categorização de grande volume de fotografias.

Todas as fotografias foram codificadas de acordo com os aspectos relacionados à limpeza, ambiência, mobiliário, condições de iluminação e ventilação, espaços terapêuticos, dimensionamento e acessibilidade dos serviços.

Após a codificação, ao serem recuperadas as imagens relacionadas a cada código oferecido pelo software prosseguiu-se a discussão dos dados com base nos documentos oficiais, registros de observação feitos pelos pesquisadores da equipe, mantendo articulação com a literatura.

O projeto de pesquisa a que este trabalho está vinculado foi aprovado pelo Comitê de Ética em Pesquisa da Universidade Federal de Goiás, protocolo 303/10, conforme as orientações da Resolução 196/96 do CONEP para pesquisa com seres humanos.

Vale destacar que todos os cuidados éticos foram seguidos, desde a apresentação do projeto de pesquisa à Diretoria de Gestão do Trabalho e Educação da Secretaria Municipal de Goiânia, até o respectivo consentimento institucional. Após esta etapa, os coordenadores dos serviços foram esclarecidos também sobre os procedimentos de coleta, tendo sua participação sido legitimada pela assinatura do Termo de Consentimento Livre e Esclarecido. Houve um compromisso, por parte dos pesquisadores, em não publicar as fotos para evitar a identificação dos serviços, condição que não ocasiona prejuízos na divulgação dos resultados deste estudo.

\section{RESULTADOS E DISCUSSÃO}

Os resultados apresentam os elementos analisados com base no referencial de Donabedian(12), mais especificamente, no componente Estrutura. Estão dispostos em aspectos relacionados à área de abrangência, estrutura física e recursos humanos.

\section{Área de abrangência}

Identificou-se que, por ocasião da coleta de dados, nem todas as regiões de Goiânia contavam com CAPS, condição que contradiz com a cobertura preconizada pela PNSM. Mesmo com o expressivo crescimento do indicador de cobertura assistencial em todas as regiões do país, em Goiás, a cobertura de CAPS/100.000 habitantes apresentou o indicador de 0,50, apresentando-se abaixo da média nacional que é de $0,72^{(13)}$.

As unidades da RESM/SMS/GO são vinculadas ao SUS e funcionam como referência para grande parcela da população no atendimento psiquiátrico neste município. 
Por ser a capital do estado e concentrar mais recursos no território, recebe também usuários provenientes de outras cidades de Goiás e de outras regiões do Brasil.

É importante destacar que o município não conta com leito psiquiátrico em hospital geral e a demanda dos usuários que necessitam de internação se dá por meio de convênio entre clínicas psiquiátricas privadas e o SUS.

\section{Estrutura física}

É primordial que o funcionamento da assistência em Psiquiatria e Saúde Mental considere os aspectos relativos à estrutura física, além de materiais e equipamentos disponíveis nos serviços.

De um modo geral, os serviços que compõem a RESM/SMS/GO apresentam estrutura física comprometida. A inadequação está associada ao dimensionamento restrito dos espaços, condições de teto e parede inadequados (infiltrações, mofo, goteiras, rachaduras), iluminação imprópria e espaços mal arejados. Além disso, foi possível perceber que alguns serviços apresentam condições de limpeza insatisfatórias, falta de materiais, equipamentos e elementos que prejudicam a privacidade dos usuários e trabalhadores nas atividades que desenvolvem. Neste quesito, em especial, ressalta-se problemas relativos à acústica dos espaços destinados aos atendimentos ou à proximidade com outras instituições que geram ruídos ou têm movimentos de pessoas que interferem no espaço privativo, muitas vezes essencial e próprio dos atendimentos em Saúde Mental.

Evidenciou-se também ausência de ambientes propícios ao conforto do trabalhador de saúde e usuários, na medida em que grande parte dos mobiliários e equipamentos estão danificados e em número aquém do necessário.

A ambiência visa qualificar os ambientes de saúde, resultando na melhoria da qualidade de vida e de saúde das pessoas, além de ser uma importante ferramenta facilitadora do processo de trabalho(14).

A ambiência dos serviços de saúde segue três eixos: o espaço que garanta a privacidade e a individualidade, valorizando o ambiente (cor, cheiro, som, iluminação, dentre outros) e assegure o conforto de usuários e trabalhadores; o espaço que possibilita a subjetividade, por meio da ação e reflexão dos processos de trabalho; e o espaço que favorece o atendimento humanizado e acolhedor $^{(15)}$.

O cenário encontrado nos serviços pesquisados nem sempre é condizente com os recursos mínimos para o oferecimento da assistência de qualidade aos usuários. Foi observado que algumas acomodações não são favoráveis para a realização de consultas, dispensação de medicamento, terapias grupais e oficinas com finalidade socioeducativas. As deficiências das instalações, além de provocar descontentamentos, podem promover limitações na organização e na execução das práticas de Saúde Mental.

Tal situação reflete o quadro de saúde pública no Brasil, que caracteriza-se por inúmeras carências em várias áreas e especialidades, constituindo-se em crises no atendimento, a despeito de algumas medidas de gestores locais a fim de minimizar as consequências aos cidadãos que precisam de atendimento digno nos dispositivos oferecidos a eles. É inegável, no atual contexto, que carecemos de iniciativas certeiras por parte das esferas gestoras para garantir os princípios do SUS e as políticas públicas tão bem delineadas, mas muitas vezes difíceis de serem exequíveis.

A insatisfação com a estrutura física e material, inadequação do local, precariedade do ambiente e restrição de materiais para a realização de oficinas terapêuticas, aparecem também como fatores que dificultam as ações dos profissionais de saúde em outras localidades ${ }^{(10)}$.

Por outro lado, não é incomum que alguns profissionais tenham dificuldades em incluir nos projetos terapêuticos outros dispositivos do território como forma de reinserção do usuário na sociedade, atendendo a proposta reintegradora do modelo psicossocial. Desta maneira, por meio de suas práticas, conservam os usuários nos serviços para protegê-los dos desafios impostos pela sociedade, remetendo à ideia de nova cronicidade ${ }^{(16-17)}$.

A esse respeito, o termo "enCAPSulado" tem sido empregado para simbolizar a inexistência da relação dos CAPS com outros espaços do território. Nesta dinâmica assistencial, a terapêutica fica restrita apenas ao CAPS sem articulação com os demais equipamentos sociais, e os usuários permanecem nessas unidades de saúde sem um plano terapêutico devidamente definido, lembrando a lógica da assistência nos hospitais psiquiátricos ${ }^{(16)}$.

Os serviços substitutivos, tais como os CAPS, possuem caráter reabilitador por serem serviços territorializados, utilizando espaços na própria comunidade, fazendo com que o usuário retome sua posição frente à sociedade, por meio de um processo que envolve construção de autonomia, liberdade e cidadania(8).

Torna-se importante portanto, a planificação e execução de projetos assistenciais que otimizem os 
recursos da comunidade, ação que além de ir ao encontro dos princípios da reinserção social, na medida em que atenda às reais necessidades dos usuários dos serviços de saúde, tende a mobilizar a criação de novas e criativas estratégias e instrumentos da atenção psicossocial por meio de parcerias interinstitucionais. Em situações em que há falta de investimentos na criação de espaços junto à comunidade, há consequentemente comprometimento da terapêutica proposta pelo modelo psicossocial(16).

No âmbito da estrutura física dos serviços pesquisados, se antes, os atendimentos se concentravam essencialmente nos consultórios, observou-se que, atualmente, outros espaços e áreas comuns são aproveitados. Há evidências de que corredores, refeitórios e áreas externas têm sido utilizados, condição que não invalida qualquer ação terapêutica, mas ao contrário, mostra avanço na própria concepção do serviço de saúde(18), desde que sejam resguardados o sigilo e a privacidade das pessoas.

Vale ressaltar, que determinados serviços da RESM/SMS/GO passaram por recente reforma para adequação da estrutura física, o que resultou em melhorias para o atendimento. Foi possível verificar que alguns deles apresentam ambientes acolhedores, com ampla área de convivência e de lazer, além da boa acessibilidade.

O espaço usado como ferramenta facilitadora do processo de trabalho, favorece a otimização de recursos, o atendimento humanizado, acolhedor e resolutivo(15). A preocupação com a estrutura física não tem origem somente na necessidade de conforto pessoal para a execução das atividades cotidianas, mas principalmente no desejo de melhor atender aos usuários, de poder executar a contento as atividades programadas ${ }^{(17)}$.

As orientações do Ministério da Saúde ${ }^{(15)}$, no quesito espaço físico, definem que os CAPS devem contar, no mínimo, com salas para atividades grupais, consultórios para atividades individuais (consultas, entrevistas, terapias), espaço de convivência, oficinas, refeitório, área externa para oficinas, recreação e esportes. Evidentemente a falta de espaço físico, inadequação do local, precariedade do ambiente e restrição de materiais para a realização das oficinas, constituem entraves para o desenvolvimento da assistência(10).

O dimensionamento restrito aparece principalmente nas unidades de Geração de Renda (Gerarte), onde são desenvolvidas atividades de artesanato. Com vistas ao cumprimento de sua missão ressalta-se a importância de estrutura adequada nestes serviços, que devido sua especificidade requer espaços e recursos para o incremento de oficinas, geralmente relacionadas às artes manuais.

Questões relativas à estrutura física devem contemplar, necessariamente, quesitos relativos à acessibilidade aos serviços. Dentre as 11 unidades visitadas, seis funcionam em edificações verticais com prejuízo no acesso dos usuários, uma vez que não dispõem de condições adequadas para os portadores de necessidades especiais. Em apenas uma delas encontrouse estrutura adaptada para tais circunstâncias.

Esta realidade mostra-se divergente da recomendação do Ministério da Saúde ${ }^{(15)}$ ao destacar que as unidades de saúde devem contemplar projetos arquitetônicos que permitam a facilitação de acesso dos usuários que tenham algum tipo de limitação física. 0 acesso à unidade de saúde pode comprometer o tratamento e/ou sua continuidade. Há de se considerar que serviços muito isolados e que apresentam difícil acessibilidade apresentam retrocesso à proposta da Reforma Psiquiátrica, pois assim eram os hospitais psiquiátricos, que dantes eram afastados das cidades, a fim de se esconder o louco, tido como um empecilho à evolução da sociedade.

Como serviços de saúde de base comunitária e aberta, os CAPS do município de Goiânia apresentam portas abertas e estão devidamente identificados, atendendo plenamente as recomendações da PNSM. Neste sentido, buscando consolidar a implantação do modelo de atenção comunitária, de base extra-hospitalar todos eles possuem área física específica e independente de qualquer instituição hospitalar.

Entretanto, a totalidade dos serviços pesquisados funcionava em locais que não foram construídos especificamente para serem unidades de saúde, sendo que, até a conclusão deste estudo, apenas um CAPS possuia sede própria, e havia passado por melhorias para adequação da estrutura física.

Vale observar que a proximidade dos pesquisadores com instâncias gestoras da Divisão de Saúde Mental da Secretaria Municipal de Saúde (DSM/SMS/GO) por meio de parcerias interinstitucionais (academia/serviço) a qual legitima a inserção de atividades acadêmicas em vários serviços da RESM/SMS/GO, permite acompanhar seu processo de expansão, em atendimento à Portaria no 3.088, em 2011, que reforça a PNSM $^{(5)}$.

Neste sentido, há de se ressaltar que a configuração da respectiva rede de serviços está diferente do cenário encontrado por ocasião do desenvolvimento desta investigação, tendo sido inaugurados dois CAPS, os quais doravante funcionam em instalações próprias (um deles 
recém criado e outro com mudança de local), além das transformações de alguns já existentes em CAPS tipo III.

Oferecer atendimento em casa alugada parece indicar uma condição provisória de funcionamento, o que não condiz com a proposta dos serviços substitutivos ser definitiva(10). Instalar-se em sede própria permite que os agentes utilizem espaços do próprio território, além de favorecer o vínculo profissional-usuário, aspecto importante do projeto terapêutico. Por outro lado, há de se considerar que funcionar em imóveis alugados não deveria invalidar o modelo de atenção psicossocial nem prejudicar a assistência.

Conforme mencionado, Goiânia conta com um Pronto Socorro Psiquiátrico (PSP), que atende somente situações de emergência e urgência da especialidade com demanda de usuários não apenas deste município. Está funcionando em um prédio anexo a uma clínica psiquiátrica da rede privada e suas acomodações ainda remetem às instituições asilares, com grades e dispositivos que reproduzem o modelo de reclusão.

Há recintos para a administração e assistência (área de acolhimento, consultórios médicos, de psicologia, sala de serviço social, sala para atividades de arteterapia, sala de medicação e farmácia), além de duas alas de internação, com enfermarias femininas e masculinas. A estrutura física é precária, com mobiliário antigo e sem manutenção, condição que levou à desativação de alguns quartos por não apresentarem condições de uso. O PSP possui também uma enfermaria com seis leitos para atendimento de intercorrências, especialmente em casos de usuários que dão entrada em franco surto psicótico e/ou agitação psicomotora. Todas as enfermarias são isoladas por grades e cadeados. A despeito de ter ampla estrutura física com espaços abertos e parte coberta, o estado de conservação não é adequado para que sejam aproveitados como área de convivência e realização de atividades de lazer, recreação ou esporte, consideradas todas favoráveis e necessárias à sua especificidade de atendimento.

Outro aspecto observado nesse serviço refere-se também às condições precárias da sala administrativa onde se armazenam os prontuários: sinais de infiltração, goteiras, fiação exposta, odor forte de mofo, além da ausência de arquivos próprios para arquivamento desses documentos, uma vez que são acondicionados em caixas de papelão. Tais condições são insalubres e comprometem a segurança de usuários, familiares e trabalhadores.

O cenário encontrado permite afirmar que O PSP apresenta condições estruturais que comprometem a assistência, à medida que não oferece conforto, nem tampouco condições para o desenvolvimento de atividades terapêuticas condizentes às necessidades dos usuários do serviço, dos trabalhadores, sendo desfavorável à interação entre eles, premissa especialmente importante num serviço de Saúde Mental. Com relação ao modelo assistencial proposto pela PNSM, de igual forma, esta unidade de saúde contrasta frontalmente com sua ideia central, pois conserva os resquícios de características manicomiais.

\section{Equipe}

Considerando o referencial teórico de avaliação em serviços de saúde adotado neste estudo, o qual envolve no conceito de estrutura aspectos relativos a recursos humanos, abrangendo número e qualificação(12), foi possível ter um panorama da composição das equipes que atuam na RESM/SMS/GO. Os resultados encontrados dão visibilidade ao quadro funcional dos profissionais lotados nos serviços, especialmente quanto à caracterização quantitativa e de formação acadêmica.

Todas as unidades de saúde contam com equipe mínima, conforme exigências legais que tratam das modalidades de CAPS e recursos humanos mínimos e da composição da equipe multiprofissional de ambulatórios especializados e serviços de urgência psiquiátrica(19-20).

Verificou-se que as equipes são compostas por diferentes categorias profissionais e por distintas áreas do conhecimento. Além de médicos psiquiatras, psicólogos e enfermeiros, fazem parte dos recursos humanos da RESM/SMS/GO arteterapeutas, artistas plásticos, assistentes sociais, clínicos gerais, educadores físicos, farmacêuticos, fonoaudiólogos, musicoterapeutas, nutricionistas, pedagogos, profissionais das artes cênicas, terapeutas ocupacionais, além de outros profissionais de nível médio.

Tal diversidade na composição das equipes é um fator que diferencia a RESM/SMS/GO da grande maioria dos serviços de Saúde Mental do Brasil que, invariavelmente, contam apenas com profissionais de saúde. Esta situação é um fator bastante favorável para a efetivação do modelo de atenção à saúde mental, com vistas a ampliar as possibilidades terapêuticas por meio do olhar multidisciplinar, na perspectiva da desejada integralidade das ações de saúde ${ }^{(4)}$.

Com relação ao quantitativo de profissionais que trabalham nas unidades especializadas de Goiânia, no período em que o estudo foi desenvolvido, encontrou-se que a maioria é representada por médicos (12,15\%), seguida de psicólogos (10,31\%), 6,26\% são assistentes sociais, $3,50 \%$ enfermeiros e $1,84 \%$ terapeutas 
ocupacionais. O percentual restante é distribuído entre as demais categorias citadas.

A realidade deparada nos locais visitados está em acordo com a tendência das equipes não ficarem mais restritas às profissões tradicionais no campo da saúde, como a enfermagem, a psicologia e a área médica(18).

\section{CONCLUSÃO}

A análise dos resultados gerados, permite afirmar que a Rede Especializada em Saúde Mental da Secretaria Municipal de Saúde de Goiânia-Goiás apresenta variedade quanto a sua estrutura, no referente à área de abrangência, componentes físicos e estruturais dos serviços, disponibilidade de materiais e equipamentos, bem como no quantitativo de recursos humanos e sua formação profissional.

O município carece de investimentos na criação de novos serviços, não só em quantidade, mas também em unidades com estruturas adequadas e compatíveis com o modelo de atenção preconizado pela PNSM. O fato de a maioria dos serviços da RESM/SMS/GO funcionar em prédios que não foram planejados para o desenvolvimento de atividades específicas compromete a assistência, além de estarem com suas estruturas físicas precárias, dificultam que as equipes implementem projetos terapêuticos de qualidade.

Por outro lado, há de se considerar que, isoladamente, a estrutura não deveria ser impedimento para a excelência no atendimento em Saúde Mental, uma vez que a assistência depende também das ações empreendidas pelos profissionais. Cabe lembrar que este estudo não teve como finalidade avaliar a dinâmica dos serviços e suas implicações com a disponibilidade de recursos físicos e materiais.

No entanto, o panorama encontrado reforça a necessidade de maior investimento em projetos estruturais que visem a melhoria do funcionamento dos serviços, adequação das estruturas existentes, na contratação e qualificação das equipes para que sejam minimamente compatíveis com as políticas públicas de saúde no país.

Reestruturar as instalações pode favorecer as condições laborais dos trabalhadores, assim como segurança e conforto aos usuários em todas as suas necessidades existenciais: físicas, psíquicas, biológicas e sociais, trazendo implicações diretas na qualidade das ações oferecidas pela rede de serviços especializados.

Particularmente para atender a PNSM, no que diz respeito à reinserção social dos usuários recomenda-se que os serviços contemplem em seus projetos terapêuticos os equipamentos sociais que o território oferece. Esta articulação fortalece os vínculos interinstitucionais além de contribuir para a resolubilidade das práticas assistenciais.

Destaca-se a importância da criação de projetos arquitetônicos que atentem para a localização dos serviços nas comunidades, de fácil acesso, com detalhamentos próprios à acessibilidade para portadores de necessidades especiais, contemplando espaços que favoreçam o atendimento em consultórios ou em locais próprios para grupos, espaços de lazer e convivência, de modo a otimizar as várias práticas terapêuticas oferecidas pelos serviços.

Por fim, se por um lado a estrutura dos serviços da RESM/SMS/GO carece de certos investimentos, é relevante salientar o seu diferencial quanto à disponibilidade dos seus recursos humanos e a heterogeneidade das equipes multidisciplinares, uma das condições para a implementação da excelência da assistência alicerçada no modelo de atenção psicossocial.

Há de se ponderar que a tarefa de proteger e melhorar a área da Saúde Mental é complexa e envolve múltiplas ações, de naturezas variadas e empreendidas por órgãos institucionais distintos. Desenvolver esta investigação foi uma delas, na medida em que permitiu sinalizar alguns indicativos do processo de avaliação da estrutura de serviços de Saúde Mental da RESM/SMS/GO, os quais possibilitaram dar visibilidade à realidade loco-regional. Num momento em que os municípios do Estado de Goiás encontram-se em pleno processo de adequação dos serviços, procurando atender os princípios da Reforma Psiquiátrica brasileira, foi essencial o conhecimento do seu cenário de práticas a fim de mobilizar esforços para o enfrentamento das dificuldades e potenciar as condições favoráveis existentes.

Os resultados obtidos neste estudo podem fornecer subsídios para análise mais ampliada do contexto assistencial brasileiro que, a despeito das diferenças entre as regiões do Brasil, também verificadas na distribuição e características dos serviços de saúde, há semelhanças, especialmente se considerar que a criação de serviços substitutivos em atenção à PNSM é recente, gerando intensas mudanças no modelo de atenção em saúde mental. Neste panorama, vive-se atualmente um momento de ajustes, buscando também articulação com outros setores da sociedade, visto que todo o território nacional passa por adequações na estruturação dos serviços, na qualificação dos recursos humanos, a fim de oferecer dignidade e resolutividade às pessoas que precisam de cuidados especializados. 
A perspectiva é contribuir para a implementação efetiva das políticas públicas da área, no sentido de atingir a excelência nos serviços de saúde mental, por meio do fortalecimento de parcerias interinstitucionais, a exemplo da estabelecida entre o grupo de pesquisa do qual os pesquisadores deste estudo fazem parte e os profissionais e gestores da esfera gestora municipal e estadual de saúde de Goiás.

Esta parceria tem rendido desdobramentos importantes respeitando aos interesses e especificidades

\section{REFERÊNCIAS}

1. World Health Organization. The world Health Report, Mental Health: New Understading, New Hope. Geneva: World Health Organization; 2001.

2. Carvalho AL, Amarante P. Avaliaçäo de qualidade dos novos serviços de saúde mental: em busca de novos parâmetros. Saúde debate. 1996;(52):74-82.

3. Cavalcanti MT. A Reforma Psiquiátrica brasileira: ajudando a construir e fortalecer o Sistema Único de Saúde. Cad Saude Publica [Internet]. 2008 [acesso em: 31 mar 2014];24(9):19623. Disponível em: http://dx.doi.org/10.1590/S0102311X2008000900001.

4. Ministério da Saúde. Reforma Psiquiátrica e política de saúde mental no Brasil. Documento apresentado à Conferência Regional de Reforma dos Serviços de Saúde Mental: 15 anos depois de Caracas [Internet]. Brasília (Brasil): OPAS; 2005 [acesso em: 31 mar 2014]. Disponível em:

http://bvsms.saude.gov.br/bvs/publicacoes/Relatorio15 anos Ca racas.pdf.

5. Portaria N. 3.088, de 23 de dezembro de 2011 (BR)

[Internet]. Institui a Rede de Atenção Psicossocial para pessoas com sofrimento ou transtorno mental e com necessidades decorrentes do uso de crack, álcool e outras drogas, noâmbito do Sistema Único de Saúde. Diário Oficial da União. 26 dez 2011 [acesso em: 31 mar 2014]. Disponível em: http://bvsms.saude.gov.br/bvs/saudelegis/gm/2011/prt3088_23 _12_2011.html.

6. World Health Organization Assessment Instrument for Mental Health Systems. WHO-AIMS report on mental health system in Brazil. A report of the assessment of the mental health system in Brazil using the World Health Organization - Assessment Instrument for Mental Health Systems (WHO-AIMS) [Internet] Brasília: WHO; 2007 [acesso em: 31 mar 2014]. Disponível em: http://www.who.int/mental health/evidence/who aims report b razil.pdf.

7. Kantorski LP, Coimbra VCC, Silva ENF, Guedes AC, Cortes JM, Santos F. Avaliação qualitativa de ambiência num Centro de Atenção Psicossocial. Cien Saude Colet [Internet]. 2011 [acesso em: 31 mar 2014];16(4):2059-66. Disponível em:

http://dx.doi.org/10.1590/S1413-81232011000400005.

8. Mielke FB, Kantorski LP, Jardim VMR, Olschowsky A, Machado MS. O cuidado em saúde mental no CAPS no entendimento dos profissionais. Cien Saude Colet [Internet]. 2009 [acesso em: 31 mar 2014];14(1):159-64. Disponível em:

http://dx.doi.org/10.1590/S1413-81232009000100021.

9. Nascimento AF, Galvanese ATC. Avaliação da estrutura dos centros de atenção psicossocial do município de São Paulo, SP. Rev Saude Publica [Internet]. 2009 [acesso em: $31 \mathrm{mar}$ 2014];43 Supl. 1:8-15. Disponível em:

http://dx.doi.org/10.1590/S0034-89102009000800003. 10. Rézio LA, Oliveira AGB. Equipes e condições de trabalho nos centros de atenção psicossocial em Mato Grosso. Esc. Anna Nery [Internet]. 2010 [acesso em: 31 mar 2014];14(2):346-54. Disponível em: http://dx.doi.org/10.1590/S1414$\underline{81452010000200019}$. das partes, a qual tem possibilitado empreender várias ações no âmbito da academia e do serviço, instâncias que bem articuladas podem gerar o impacto desejado na assistência aos usuários dos serviços especializados.

Vale dizer que o tema referente à avaliação de serviços, especialmente de serviços em saúde mental, tem despertado também o interesse de outros grupos de pesquisadores no Brasil, diante da premente necessidade de se criar indicadores de avaliação que atendam às especificidades da área.

11. Wetzel C, Kantorski LP. Avaliação de serviços em saúde mental no contexto da reforma psiquiátrica. Texto contexto enferm [Internet]. 2004 [acesso em: 31 mar 2014];13(4):543-8. Disponível em: http://dx.doi.org/10.1590/S0104-

07072004000400012.

12. Donabedian A. Explorations in quality assessment and monitoring. Michigan: Health Administration Press; 1980. 13. Ministério da Saúde. Saúde Mental em Dados-10, Ano VII, n. 10, março de 2012. Brasília (Brasil): Ministério da Saúde; 2012 [acesso em: 31 mar 2014]. Disponível em:

http://saudeecosol.files.wordpress.com/2012/03/saude-mentalem-dados-10-ms.pdf.

14. Olschowsky A, Glanzner CH, Mielke FB, Kantorski LP, Wetzel C. Avaliação de um Centro de Atenção Psicossocial: a realidade em Foz do Iguaçu. Rev Esc Enferm USP [Internet]. 2009 [acesso em: 31 mar 2014];43(4):781-7. Disponível em:

http://dx.doi.org/10.1590/S0080-62342009000400007.

15. Ministério da Saúde. Secretaria de Atenção à Saúde. Ambiência [Internet]. $2^{a}$ ed. Brasília (Brasil): Ministério da Saúde; 2010 [acesso em: 31 mar 2014]. Disponível em: http://bvsms.saude.gov.br/bvs/publicacoes/ambiencia 2ed.pdf. 16. Wetzel C, Kantorski LP, Olschowsky A, Schneider JF, Camatta MW. Dimensões do objeto de trabalho em um Centro de Atenção Psicossocial. Cien Saude Colet [Internet]. 2011 [acesso em: 31 mar 2014];16(4):2133-43. Disponível em: http://dx.doi.org/10.1590/S1413-81232011000400013.

17. Guimarães JMX, Jorge MSB, Assis MMA. (In)satisfação com o trabalho em saúde mental: um estudo em Centros de Atenção Psicossocial. Cien Saude Colet [Internet]. 2011 [acesso em: 31 mar 2014];16(4):2145-54. Disponível em:

http://dx.doi.org/10.1590/S1413-81232011000400014.

18. Pinho LB, Rodrigues J, Kantorski LP, Olschowsky A, Schneider JC. Desafios da prática em saúde mental na perspectiva do modo psicossocial: visão de profissionais de saúde. Rev. Eletr. Enf. [Internet]. 2012 [acesso em: 31 mar 2014];14(1):25-32. Disponível em: http://dx.doi.org/10.5216/ree.v14i1.14352. 19. Portaria GM no 336, de 19 de fevereiro de 2002. Estabelece que os Centros de Atenção Psicossocial poderão constituir-se nas seguintes modalidades de serviços: CAPS I, II, III, definidos por ordem crescente de porte/complexidade e abrangência populacional, conforme disposto nesta portaria. Diário Oficial da União. 20 fev 2002.

20. Portaria $n^{\circ} 224 / M S$, de 29 de janeiro de 1992. Institui diretrizes e normas para o Atendimento Ambulatorial/Hospitalar em Saúde Mental. Diário Oficial da União. 30 jan 1992.

Artigo recebido em 10/09/12.

Aprovado para publicação em 07/10/13.

Artigo publicado em 31/03/2014. 